\title{
GaAs-BASED WAVEGUIDE MOEMS
}

\author{
O. Blum Spahn, T. Bakke, C. P. Tigges, J. Johnson, G. Grossetete, F. R. Gass, E. M. Ochoa*, J. L. Reno, J. \\ F. Klem, G. M. Peake, C. T. Sullivan
}

Sandia National Laboratories, MS0603, Albuquerque, NM 87185

Phone: (505) 844-3458, Fax: (505) 844-8985, Email: oblum@ sandia.gov

*Air Force Institute of Technology, Wright-Patterson AFB, OH

\begin{abstract}
We describe our work on GaAs-based waveguide switch that is also a mechanical cantilever. Several types of $1 \mathrm{xn}$ switches have been fabricated and performance of a $1 \times 2$ device is presented. Switching voltages between $6 \mathrm{~V}$ and $13 \mathrm{~V}$ volts and switching speeds as low as $32 \mu \mathrm{sec}$ have been measured.
\end{abstract}

\section{INTRODUCTION}

Recently MOEMS have undergone a period of explosive growth, both in terms of academic and commercial interest. While silicon has played a dominant role, there is an increasing interest in compound semiconductor approaches, driven largely by emerging broadband communication and sensor applications.

Use of compound semiconductors for realization of MEMS and MOEMS has several advantages. Due to epitaxial growth, they are monocrystalline, atomically flat and have extremely well controlled thickness and stress. Furthermore, use of direct band gap semiconductors allows for incorporation of optical functionality into mechanical structures, for example truly monolithic integration of lasers, MEMS and detectors. In addition, there are numerous combinations of materials and release etches which can result in MEMS/MOEMS structures [1]. Finally, the zinc blende structure allows for piezoelectricity as a result of lack of center of symmetry (in contrast to silicon), which leads to interesting sensing applications. In this paper we describe $1 \times 2$ and $1 \times 4$ switch consisting of a waveguide embedded in a cantilever, fabricated in GaAs-based materials. Possible applications include a true-time delay for low power, wide-bandwidth, space-borne phased array antenna, nonvolatile mechanical memory with latching waveguide switches and a number of sensing applications, such as 3-axis accelerometer. $\mathrm{f}$ integrated with active sources and detectors, such devices can be used for detection of small displacements, as well as implementation of integrated multiwavelength laser sources for wavelength division multiplexing (WDM).

\section{EXPERIMENTAL DETAILS}

The 1x2 switch layout and layer cross section of the waveguide are shown schematically in Figure $1 \mathrm{a}$ and $\mathrm{b}$. Fabrication process consists of contact metal patterning, waveguide patterning and dry etch, wet chemical release etching and sublimation drying. Scanning electron micrograph of a completed waveguide is shown in Figure 2. Actuation is accomplished by electrostatic means, by application of bias between the movable waveguide and static electrodes. This results in $4 \mu \mathrm{m}(12 \mu \mathrm{m})$ deflection of the $1 \times 2(1 \times 4)$ cantilevered waveguide in the plane of the wafer, which lines it up with one of the two output waveguides.
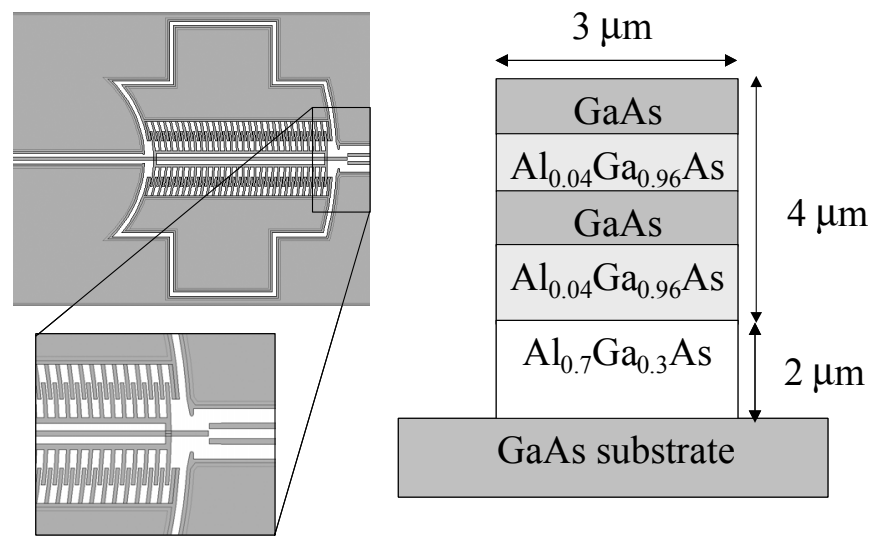

Figure 1 a) schematic layout of a 1x2 switch with the input/output section shown in the inset $b$ ) layer structure of the $3 \mu \mathrm{m}$ wide and 4 um tall waveguide

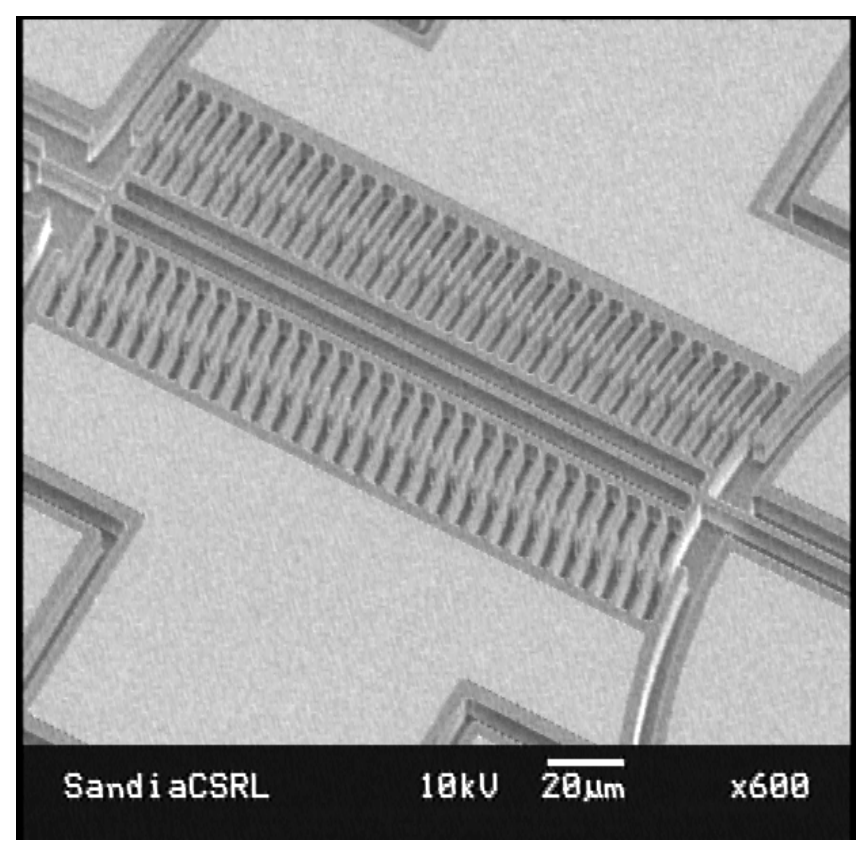

Figure 2 A scanning electron micrograph (SEM) of a top view of fabricated $1 \times 2$ switch. 
We also have fabricated a 1x8 waveguide with a $28 \mu \mathrm{m}$ deflection. 1x2 waveguide cantilevers switch at 6-13 V with resonant frequencies between $2 \mathrm{kHz}$ and $4 \mathrm{kHz}$ for beam lengths between $450 \mu \mathrm{m}$ to $700 \mu \mathrm{m}$ as shown in Figure 3. Resonant frequency and $\mathrm{Q}$ were measured by DC biasing the movable cantilever to a point where one half of the maximum optical power is observed in the corresponding output waveguide. Then the AC perturbation was applied and temporal power oscillations were a measured at the output waveguide. Resonant frequency and $\mathrm{Q}$ were obtained from the frequency of the oscillations and their damping.

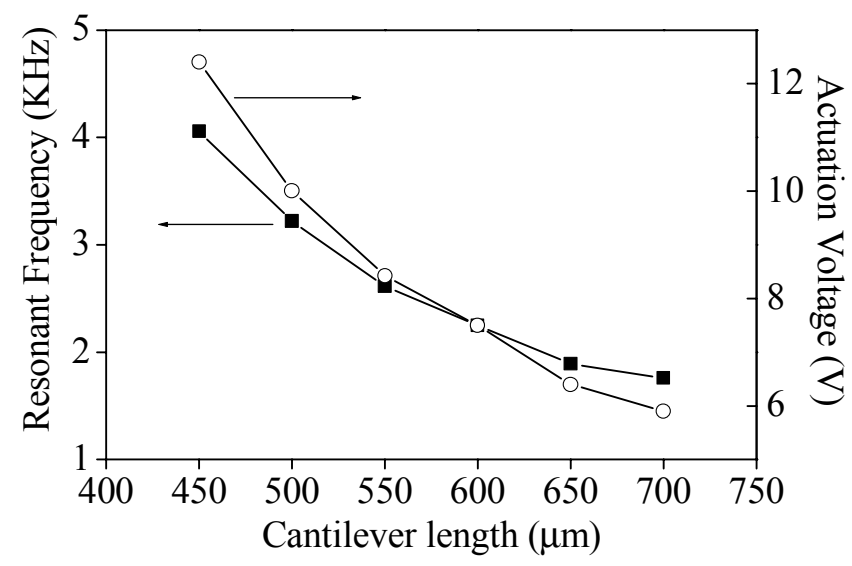

Figure 3 Actuation voltages (voltage for maximum coupling into the output waveguide) for a $1 \times 2$ switch for as a function of the cantilever length.

Measured Q values fall between 9 and 4. All measurements are taken in ambient atmosphere. Figure 4 shows optical power measured at the output waveguide as a function of applied voltage for a $1 \times 2$ and a $1 \times 4$ switch as the cantilever is deflected in one direction.

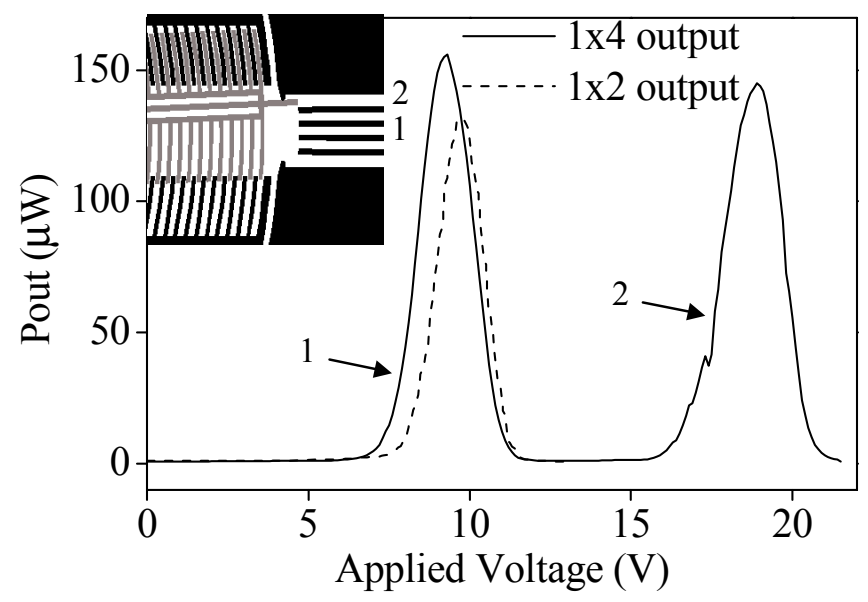

Figure 4 Optical power for a 1x4 (solid line) and 1x2 (dashed line) switch at the output waveguide as a function of bias voltage which deflects the input cantilever waveguide in and out of alignment with the fixed output waveguides (in one direction). Inset shows a schematic of a 1x4 switch deflected in one direction past the output waveguide \#1 and \#2. Output power in these waveguides is indicated on the plot with a waveguide number.

Waveguides are designed for single mode operation at 1.32 $\mu \mathrm{m}$, which has been confirmed experimentally for appropriate launch conditions. Experimentally measured end-to-end loss was found to be on the average $0.8 \mathrm{~dB} / \mathrm{cm}$ excluding fiber to waveguide insertion loss. Major contributors to the loss consist of cross through loss $\sim 0.3 \mathrm{~dB}$ and undercut interface loss $<0.05 \mathrm{~dB}$ (loss due to transition from undercut region of the waveguide to the anchored region). These optical results were obtained from a previous generation of waveguides [2] using thermally detuned Fabry-Perot method. Optical measurements on the current generation are ongoing. It should be noted that the air gap between the movable cantilever waveguide and the fixed output waveguide is designed to be resonant and thus to minimize transmission losses. Dependence of loss on the size of the air gap is described elsewhere [3].

Switching speed of these types of waveguides has been predicted to be less than $100 \mu \mathrm{sec}$. In fact, previous generation of device was measured to switch at $44 \mu \mathrm{sec}$ for a $350 \mu \mathrm{m}$ long cantilever. Use of an appropriate accelerate-brake voltage scheme on the opposite stator electrodes further reduced the switching speed to $32 \mu \mathrm{sec}$.

\section{CONCLUSIONS}

While there have been reports of silicon-based MOEMS waveguides [4], our work represents a novel approach in utilization of compound semiconductors which through their unique properties allow for innovative applications, particularly in the area of monolithically integrated MEMS and photonics

\section{ACKNOWLEDGMENTS}

Sandia National Laboratories is a multiprogram laboratory operated by Sandia Corporation for the United States Department of Energy under contract No. DE-AC04-94AL85000.

\section{REFERENCES}

1. K. Hjort, "Sacrificial etching of III-V compounds for micromechanical devices", J. Micromech. Microeng, 370, 6 (1996)

2. O. B. Spahn, C. T. Sullivan, T. Bakke, A. Allerman, J. Reno, G. Grossetete, J. Lean, C. Fuller, "Promise and progress of GaAs MEMS and MOEMS", 2001 International Conference on Compound Semiconductor Manufacturing Technology, 21-24 May 2001, Las Vegas, NV, USA, p.77-80

3. T. Bakke, C. P. Tigges, J. J. Lean, C. T. Sullivan and O. B. Spahn, "Planar Microoptomechanical Waveguide Switches", IEEE J. Select. Topics in Quantum Electron.” 64, 8(2002)

4. E. Ollier, P. Mottier "Integrated electrostatic micro-switch for optical fibre networks driven by low voltage", Electron. Lett., 2007, 32 (1996) 